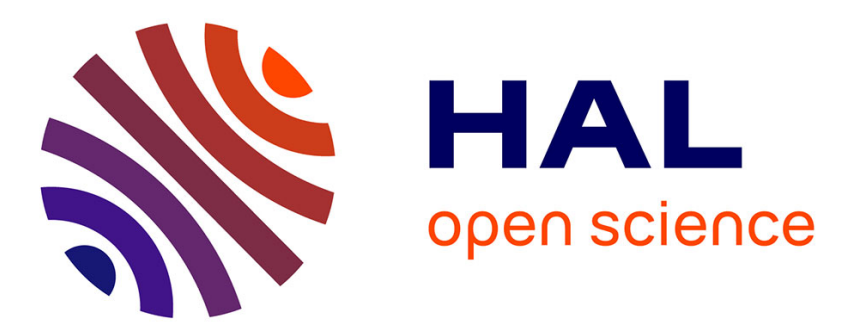

\title{
EFFECT OF CONTACT VISCOSITY AND ROUGHNESS ON INTERFACE STIFFNESS AND WAVE PROPAGATION
}

\author{
Anil Misra, Orestes Marangos
}

\section{- To cite this version:}

Anil Misra, Orestes Marangos. EFFECT OF CONTACT VISCOSITY AND ROUGHNESS ON INTERFACE STIFFNESS AND WAVE PROPAGATION. 35th Annual Review of Progress in Quantitative Nondestructive Evaluation Univ Chicago, Chicago, Jul 2008, Chicago, United States. pp.105-112. hal-00555518

\author{
HAL Id: hal-00555518 \\ https://hal.science/hal-00555518
}

Submitted on 13 Jan 2011

HAL is a multi-disciplinary open access archive for the deposit and dissemination of scientific research documents, whether they are published or not. The documents may come from teaching and research institutions in France or abroad, or from public or private research centers.
L'archive ouverte pluridisciplinaire HAL, est destinée au dépôt et à la diffusion de documents scientifiques de niveau recherche, publiés ou non, émanant des établissements d'enseignement et de recherche français ou étrangers, des laboratoires publics ou privés. 


\title{
EFFECT OF CONTACT VISCOSITY AND ROUGHNESS ON INTERFACE STIFFNESS AND WAVE PROPAGATION
}

\author{
Anil Misra $^{1}$ and Orestes Marangos ${ }^{1}$ \\ ${ }^{1}$ Civil, Environmental and Architectural Engineering Department, The University of \\ Kansas, Lawrence, KS 66045
}

\begin{abstract}
The authors have developed a micromechanical methodology for modeling contact behavior of rough solids. This methodology has been applied to determine complexvalued effective normal and shear stiffnesses of interfaces by considering surface roughness, and viscous asperity contact force laws. In this work, the micromechanically derived complex effective stiffnesses are used in conjunction with the imperfectly bonded interface model to perform a parametric study by varying surface roughness, contact viscosities, and frequency of the incident wave. We find that the surface roughness and asperity properties compete in determining the stiffness behavior, and consequently, the wave propagation behavior of rough interfaces.
\end{abstract}

Keywords: Acoustics, Interfaces, Reflection, Transmission, Effective Stiffness.

PACS: 43.20.El, 43.25.Ts

\section{INTRODUCTION}

The concept of imperfectly bonded interface models has been widely used to investigate plane wave propagation through contacts between two rough solids [see for example 1-3]. In these models, the displacement discontinuity at the interface is accommodated through effective interface stiffnesses. In the recent past, the authors have developed a micromechanical methodology for determining the effective interface stiffness [4-6]. This methodology leads to complex-valued effective stiffness that incorporates the effects of surface roughness and rate-dependent nature of asperity contacts [7].

In this work, the micromechanically derived complex effective stiffnesses are used in conjunction with the imperfectly bonded interface model to perform a parametric study by varying surface roughness, contact viscosities, and frequency of the incident wave. We find that the surface roughness and asperity properties compete in determining the overall behavior of the interface. For example, the overall relaxation times of the interface depend upon the roughness as well as the contact normal and shear relaxation times. Furthermore, these overall relaxation times are different in the normal and shear directions. These findings have critical implication on the interpretation of wave propagation through rough interfaces in terms of energy dissipation and phase lag of transmitted and reflected waves.

In the subsequent discussion, we first briefly describe the derivation of the effective interface stiffness. We then utilize the effective stiffness in the imperfectly bonded 
interface model to perform a parametric study of the behavior of wave transmission and reflection.

\section{COMPLEX-VALUED EFFECTIVE INTERFACE STIFFNESS}

In the micromechanical methodology, we explicitly consider the contact between asperities of the rough surfaces. The stress-deformation relationship for an interface is then derived by utilizing: (1) the distribution functions of asperity heights and contact orientations, and (2) the overall kinematic constraints and equilibrium conditions [4-6]. In this paper, we give a brief derivation for linear rate-dependent interface behavior that can be used in the imperfect interface model to study wave propagation. Needless to say, for such interfaces a rate-dependent asperity contact behavior need to be considered.

\section{Asperity Behavior and Interface Roughness}

For this work, we assume a simple Kelvin-Voigt rheological model to describe the asperity behavior [7]. In this case, the asperity contact forces, $f_{i}^{c}$, are related via the asperity contact stiffnesses, $K_{i j}{ }^{c}$, and asperity contact viscosities, $\eta_{i j}{ }^{c}$, to asperity contact displacements and displacement rates as follows

$$
f_{i}^{c}=K_{i j}^{c} \delta_{j}^{c}+\eta_{i j}^{c} \dot{\delta}_{j}^{c}
$$

Although the asperity contact stiffnesses and viscosities depend upon the contact loading condition, loading independent stiffnesses and viscosities can be used to illustrate the essence of the modeling methodology as well as the impact on wave propagation behavior. For further discussion, it is convenient to express the asperity stiffness tensor, $K_{i j}{ }^{c}$, in terms of asperity stiffness that describes the behavior along the direction of normal and tangent to an asperity contact, such that

$$
K_{i j}^{c}=K_{n}^{c} n_{i}^{c} n_{j}^{c}+K_{s}^{c}\left(s_{i}^{c} s_{j}^{c}+t_{i}^{c} t_{j}^{c}\right)
$$

where $K_{n}{ }^{c}$ and $K_{s}{ }^{c}$ denote nonlinear asperity stiffness along the normal and tangential direction of the asperity. The unit vector $\mathbf{n}$ is normal to the asperity contact surface and vectors $\mathbf{S}$ and $\mathbf{t}$ are arbitrarily chosen on the plane tangential to the asperity contact surface, such that nst forms a local Cartesian coordinate system as illustrated in Fig. 1.

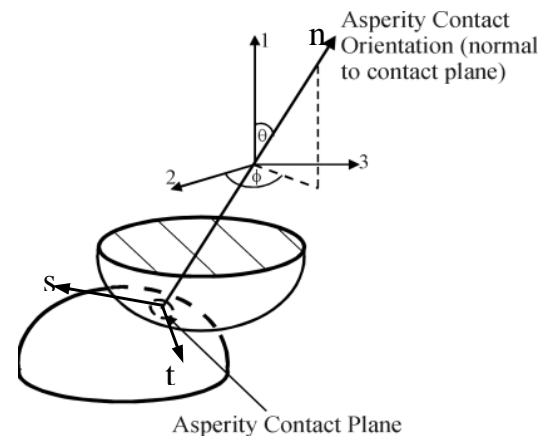

FIGURE 1. Asperity contact and the coordinate system. 
It is noted that the stiffness terms that cross-link normal and shear behavior are assumed to be negligible in accordance with the theories for contact of smooth nonconforming bodies. Similarly, the asperity viscosity tensor, $\eta_{i j}$, may be written as:

$$
\eta_{i j}^{c}=\eta_{n}^{c} n_{i}^{c} n_{j}^{c}+\eta_{s}^{c}\left(s_{i}^{c} s_{j}^{c}+t_{i}^{c} t_{j}^{c}\right)
$$

We define the following time constants for the asperity contacts:

$$
t_{n}^{c}=\frac{\eta_{n}^{c}}{K_{n}^{c}} \text { and } t_{s}^{c}=\frac{\eta_{s}^{c}}{K_{s}^{c}}
$$

Furthermore, we consider the distribution functions of asperity heights and contact orientations to find the number of asperity contacts $N_{\Omega}$ in the interval represented by solid angles $\Omega$ and $\Omega+d \Omega$, as follows:

$$
N_{\Omega}=N_{r} \xi(\Omega) d \Omega
$$

where $N_{r}$ is the total number of asperity contacts under a given interface closure, and $\xi(\Omega)$ denotes a 3-dimensional density function that describes the concentrations of asperity contact orientations [4-5]. For an interface with $N$ asperities per unit area, the total number of asperity contacts under a given interface closure, $r$, is given by:

$$
N_{r}=\int_{0}^{r} N H(r) d r
$$

where $H(r)$ represents the density function for asperity heights. The density function, $\xi(\Omega)$, of asperity contact orientations distribution in the domain: $0 \leq \theta \leq \pi / 2 a, 0 \leq \phi \leq 2 \pi$, is given by:

$$
\xi(\Omega)=\frac{a \sin a \theta}{2 \pi \sin \theta}\left[1+\frac{b}{4}(3 \cos 2 a \theta+1)\right] \quad\left(0 \leq \theta \leq \frac{\pi}{2 a} ; \quad 0 \leq \phi \leq 2 \pi ; \quad a \geq 1\right)
$$

where $\Omega$ represents the solid angle formed by $\phi$ and $\theta$ defined in Fig. 1, and parameters $a$ and $b$ determine the shape of the density function $\xi(\Omega)$. The average asperity contact angle in the meridional direction may be obtained as the expectation $E[\theta]=\int \theta \xi(\Omega) d \Omega$, given by:

$$
E[\theta]=\frac{6-b}{6 a}
$$

The density function in (7) has the ability to model surfaces with varying roughness. As discussed in [5], the asperity contacts for smooth interfaces have a greater tendency to concentrate in the direction normal to the interface than that for rough interfaces. It is noteworthy that, as parameter, $a$, increases, the contact distribution concentrates towards the direction normal to the interface. In particular, the density function, $\xi(\Omega)$, behaves like a delta function in the limit $a \rightarrow \infty$ and yields an expectation $E[\theta]=0$, which represents a concentrated contact orientation, normal to the interface of a perfectly smooth joint. In 
general, the parameter, $a$, describes the extent of the asperity contacts in the meridional direction as well as the mean asperity contact orientation. Parameter, $b$, on the other hand, describes the shape of the contact distributions within the meridional extent of asperity contacts. For example, the extent of asperity contact inclination in meridional direction is $\pi / 2$ for $a=1$ and $\pi / 4$ for $a=2$, while, the shapes of contact distributions vary with the values of parameter $b$. Parameter $b=-1$, represents an interface on which the asperity contacts tend to orient closer to the horizon, i.e. $\theta=\pi / 2$. In contrast, parameter $b=2$, represents an interface on which the preferred orientation is closer to the interface normal, i.e. $\theta=0$. For $b=0$, the asperity contacts are equally distributed in the meridional direction. Fig. 2 gives the variation of average asperity contact angle with the asperity contact orientation parameter $a$ for parameter $b=-1$ and 1 , respectively.

\section{$\underline{\text { Overall Interface Stiffness }}$}

The overall behavior of the interface can be related to the asperity behavior by considering energy balance as follows:

$$
F_{i} \Delta_{i}=N \int_{r} \int_{\Omega} f_{i}^{c} \delta_{i}^{c} \xi(\Omega) H(r) d \Omega d r
$$

where the interface overall traction $F_{i}$ is given as force per unit area since $\mathrm{N}$ is measured per unit area of an interface and $\Delta_{i}$ is the interface displacement. The overall stiffness of the interface can be derived through two approaches, referred here as (1) the kinematic approach, and (2) the static approach. Since the static approach represents interaction between asperities, we follow this approach for our further discussion. In the static approach we assume that the asperity contact force is related to the overall traction as follows:

$$
f_{i}^{c}=\frac{1}{N_{r}} F_{i}
$$

which implies that asperities are spaced close together. In this case, the overall interface displacement, $\Delta_{i}$, is obtained from the summation of the displacements, $\delta_{i}^{c}$, developed at asperities, which for a large number of asperity contacts is written as the following integral by using equations (9) and (10):

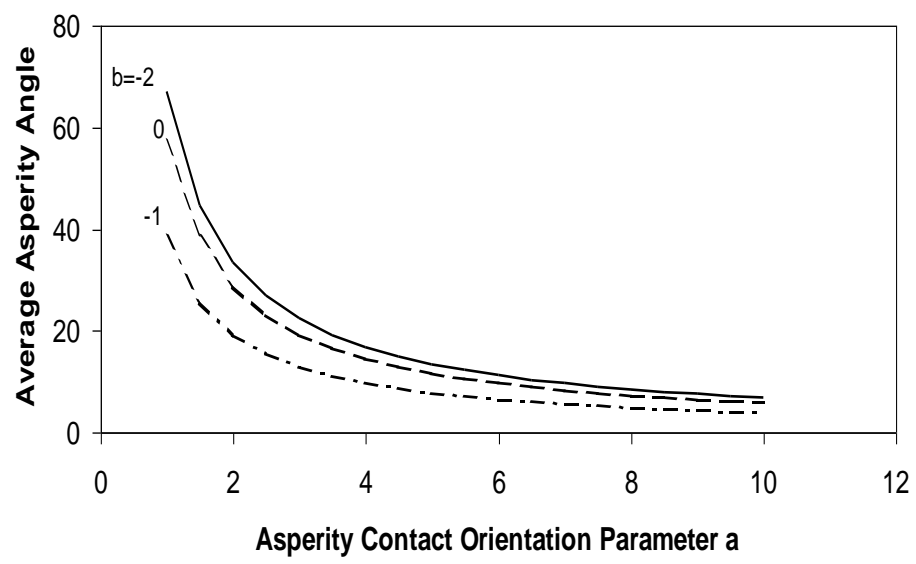

FIGURE 2. Average asperity contact angle versus asperity contact orientation parameter, $a$. 


$$
\Delta_{i}=\frac{N}{N_{r}} \iint_{r} \delta_{i}^{c} \xi(\Omega) H(r) d \Omega d r
$$

The asperity displacement, $\delta_{i}^{c}$, is obtained in terms of the asperity force, $f_{i}^{c}$, from the solution of the differential operator form of the asperity force-displacement relationship given in equation (1), as follows:

$$
\delta_{i}^{c}=\int_{0}^{t}\left(\frac{1-\exp \left(1-\frac{t}{t_{n}^{c}}\right)}{K_{n}^{c}} n_{i}^{c} n_{j}^{c}+\frac{1-\exp \left(1-\frac{t}{t_{s}^{c}}\right)}{K_{s}^{c}}\left(s_{i}^{c} s_{j}^{c}+t_{i}^{c} t_{j}^{c}\right)\right) \frac{d}{d t} f_{j}^{c} d t
$$

Thus, the overall interface displacement, $\Delta_{i}$, may be written as:

$$
\Delta_{i}=\int_{0}^{t} J_{i j} \frac{d}{d t} F_{j} d t
$$

where interface creep function, denoted by, $J_{i j}$, is obtained as the following integral for the loading independent contact behavior:

$$
J_{i j}=\frac{1}{N_{r}} \int_{\Omega}\left(\frac{1-\exp \left(1-\frac{t}{t_{n}^{c}}\right)}{K_{n}^{c}} n_{i}^{c} n_{j}^{c}+\frac{1-\exp \left(1-\frac{t}{t_{s}^{c}}\right)}{K_{s}^{c}}\left(s_{i}^{c} s_{j}^{c}+t_{i}^{c} t_{j}^{c}\right)\right) \xi(\Omega) d \Omega
$$

The integral form of the stress-deformation relationship may be used to obtain stiffness functions or complex compliances and stiffnesses. For wave propagation studies, the complex stiffnesses are of interest and, equation (13) can be recast into the following form:

$$
F_{i}(\omega)=R_{i j}^{*} \Delta_{j}(\omega)
$$

where the complex stiffness tensor, $R_{i j}{ }^{*}$, takes the following form:

$$
R_{i j}^{*}=R_{i j}(\omega)-I \bar{R}_{i j}(\omega) \text { with } R_{i j}(\omega) \geq 0 \text { and } \bar{R}_{i j}(\omega) \geq 0
$$

It is noteworthy that, the modulus of the complex stiffness determines the amplitude while the ratio of imaginary and real parts determines the phase of the interface stress when subjected to a harmonic displacement, such as an input plane wave.

\section{RESULTS FOR REFLECTION AND TRANSMISSION AT INTERFACES}

The imperfectly bonded interface model, also known as linear slip or displacement discontinuity approach [1-3], has been widely used to investigate wave propagation through interfaces between solids. At an imperfect interface between two media, tractions at upper medium $\mathrm{A}$ and lower medium $\mathrm{B}$ are continuous and displacements are discontinuous, which lead to the following equations: 


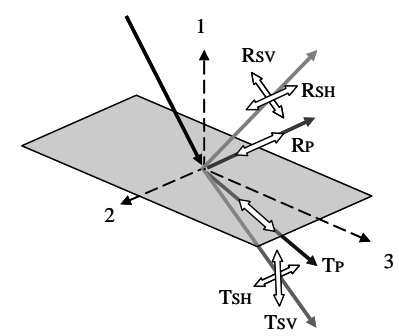

FIGURE 3. A schematic of wave reflection and transmission at an imperfect interface.

$$
\begin{gathered}
\sum_{n=0}^{3} \sigma_{i 1}^{(n) A}=\sum_{n=4}^{6} \sigma_{i 1}^{(n) B} \\
\sum_{n=4}^{6} \sigma_{i 1}^{(n) B}=\left(\sum_{n=4}^{6} u_{j}^{(n)}-\sum_{n=0}^{3} u_{j}^{(n)}\right) R_{i j}
\end{gathered}
$$

where $n=0-3$ and $n=4-6$ are the wave modes in the incident and transmitting media, respectively. For convenience, we choose a coordinate system, such that the direction of the incident wave propagation is within the 1-3 plane shown in Fig. 3. Equations (17) and (18) may be combined to obtain the amplitudes of the reflected and transmitted $\mathrm{P}$ and $\mathrm{S}$ waves given in terms of complex valued reflection, $\left\langle R_{P}, R_{S V}, R_{S H}\right\rangle$, and transmission, $\left\langle T_{P}\right.$, $T_{S V}, T_{S H}>$, coefficients, respectively. Under normal incidence of a P-wave, the amplitudes and phase of the reflected and transmitted wave are given as

$$
\begin{gathered}
\left|T_{P}\right|=\sqrt{\frac{\left(4 R_{11}{ }^{2}+2 \omega Z_{P} \bar{R}_{11}+4 \bar{R}_{11}{ }^{2}\right)^{2}+4\left(\omega Z_{P}\right)^{2} R_{11}{ }^{2}}{\left[4 R_{11}{ }^{2}+\left(\omega Z_{P}+2 \bar{R}_{11}\right)^{2}\right]^{2}}} \text { and } \phi_{P}^{T}=\arctan \left(\frac{\omega Z_{P} R_{11}}{2 R_{11}{ }^{2}+\omega Z_{P} \bar{R}_{11}+2 \bar{R}_{11}{ }^{2}}\right) \\
\left|R_{P}\right|=\sqrt{\frac{\left(\omega Z_{P}\right)^{2}}{4 R_{11}{ }^{2}+\left(\omega Z_{P}+2 \bar{R}_{11}\right)^{2}}} \text { and } \phi_{P}^{R}=\arctan \left(-\frac{2 R_{11}}{\omega Z_{P}+2 \bar{R}_{11}}\right)
\end{gathered}
$$

We use the expressions in equations (19) and (20) to illustrate the competing effect of interface roughness and asperity properties. In Figs. 4 and 5 we have plotted the transmitted and reflected wave amplitudes and corresponding phases for specific values of asperity stiffness constants computed using the following asperity properties: $N K_{n}=220$ $\mathrm{GPa} / \mathrm{m}, K_{s}=0.5 K_{n}, t_{s}=0 \mathrm{~s}$, and three values of $t_{n}=0 \mathrm{~s}, 10^{-3} \mathrm{~s}$ and $10^{-4} \mathrm{~s}$, respectively. Different interface roughnesses are modeled by considering the following values of asperity orientation parameters, $a=1,2,4$ and 8 , which correspond to the mean asperity contact orientation of $57^{\circ}, 29^{\circ}, 14^{\circ}$ and $7^{\circ}$, respectively. Finally, the following values were used for the properties of the bulk materials: shear modulus $G=23 \mathrm{GPa}$, Poisson's ratio $v$ $=0.224$, density $\rho=3 \mathrm{Mg} / \mathrm{m}^{3}$, and P-wave velocity $c_{p}=4638 \mathrm{~m} / \mathrm{s}$. Based upon this parametric study we can make the following conclusions:

- The surface roughness and asperity properties compete in determining the wave propagation behavior of the interface. For example, we can obtain similar (same or very close) reflection or transmission coefficients for a given surface roughness and different asperity viscosities.

- The surface roughness has a small (negligible) effect when the time period of the wave is smaller than the asperity relaxation time $\left(<\sim 10 t_{n}\right)$, that is waves with low frequencies or long wavelengths.

- Relatively smooth interfaces tend to have a smaller reflection and higher transmission coefficients. 
- At certain asperity contact viscosities and surface roughness (average asperity contact angle $<30^{\circ}$ ), the wave transmission behavior resembles that of a perfect (or welded) interface as seen in Fig. 4 for $t_{n}=0.001$.
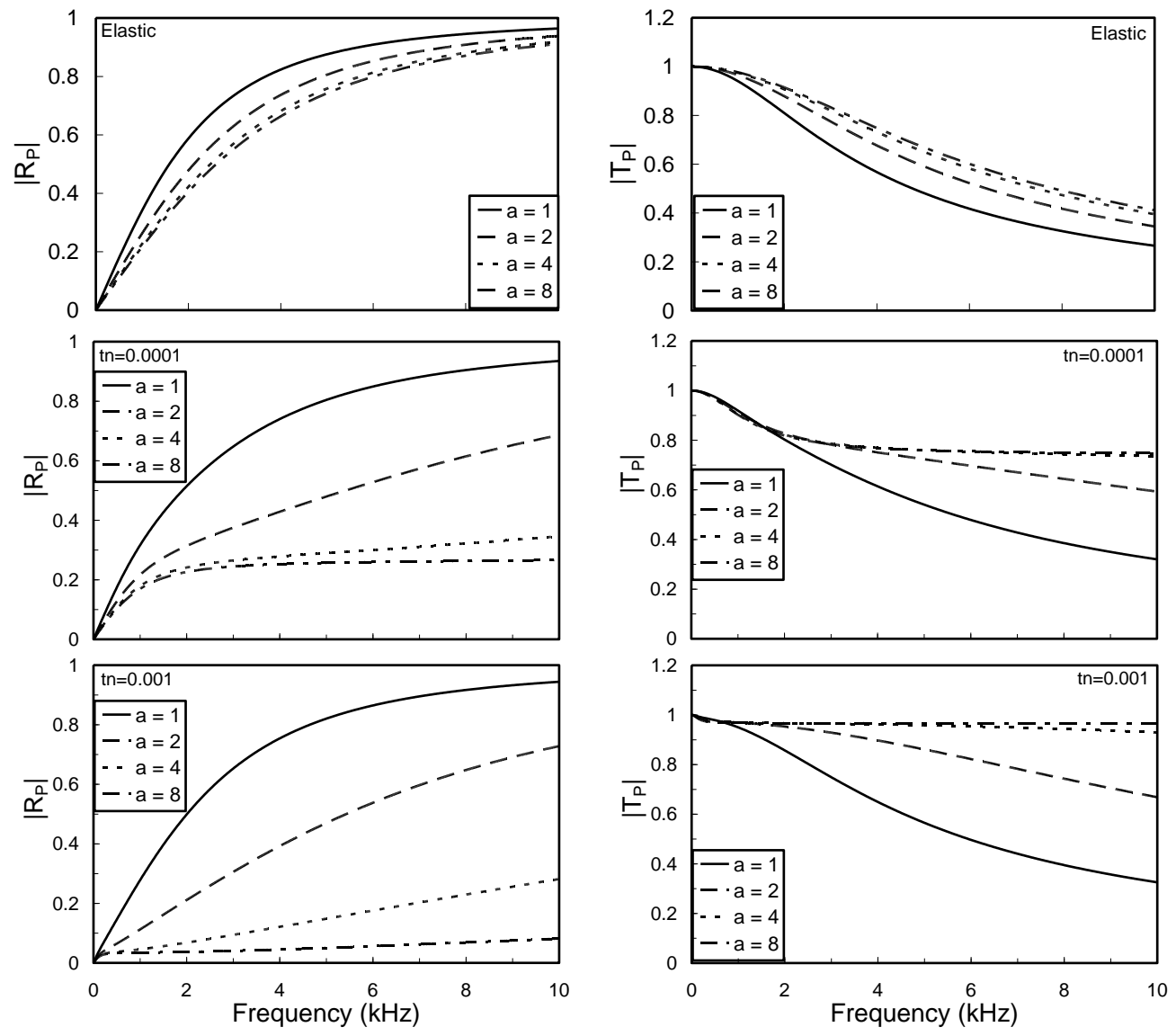

FIGURE 4. Effect of surface roughness and asperity viscosity on P-wave reflection and transmission coefficients under normal incidence and normal closure.
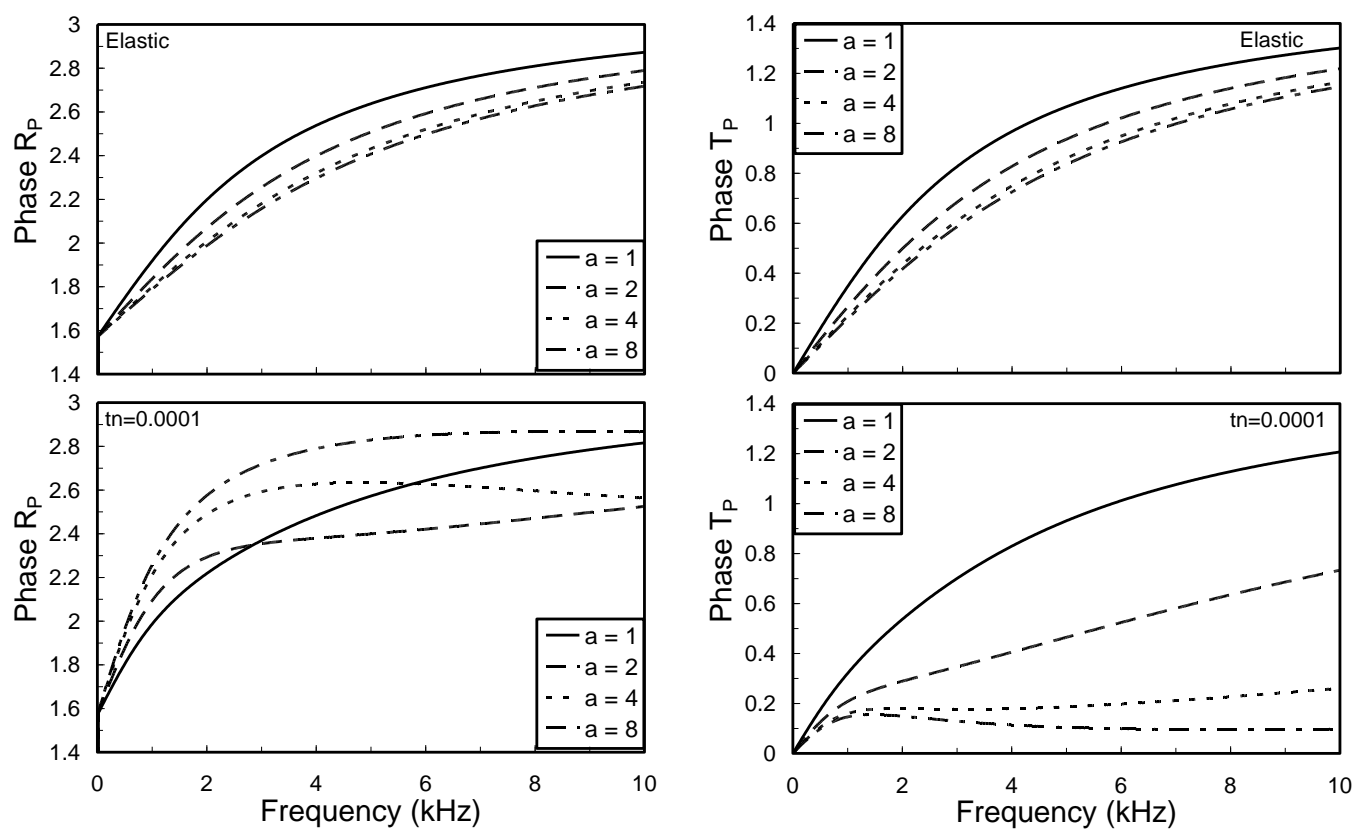

FIGURE 5. Effect of surface roughness and asperity viscosity on reflected and transmitted P-wave phases under normal incidence and normal closure. 

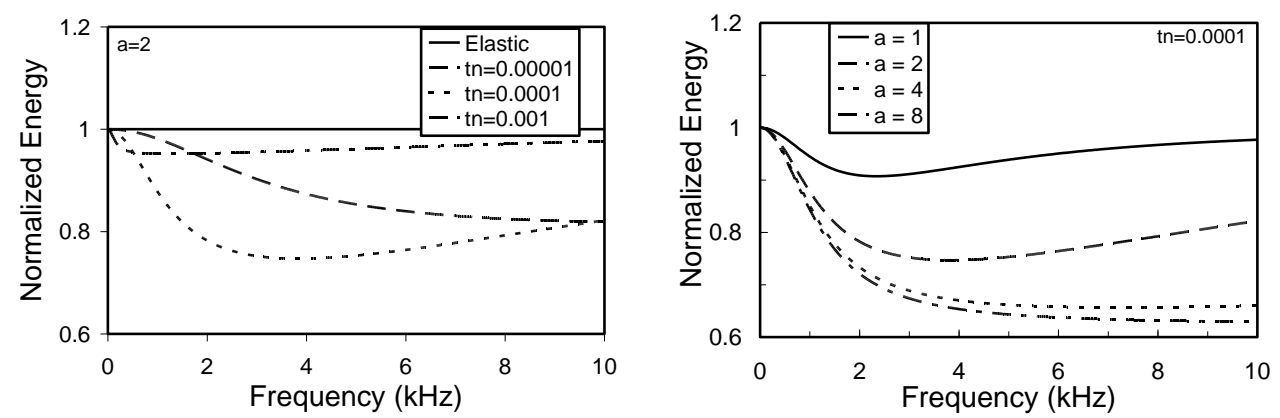

FIGURE 6. Effect of surface roughness and asperity viscosity on energy dissipation during P-wave transmission through interfaces under normal incidence.

Furthermore, we find that the wave energy dissipated in a viscoelastic interface is a function of the surface roughness and asperity viscosity. To this end, we consider the normalized specific energy rate, $E_{P}$, defined as the energy per unit area per unit time given by:

$$
E_{P}=\left|R_{P}\right|^{2}+\left|T_{P}\right|^{2}=\frac{4 R_{11}{ }^{2}+4 \bar{R}_{11}{ }^{2}+\left(\omega Z_{P}\right)^{2}}{4 R_{11}{ }^{2}+\left(\omega Z_{P}+2 \bar{R}_{11}\right)^{2}}=1-\frac{4\left(\omega Z_{P}\right)^{2} \bar{R}_{11}{ }^{2}}{4 R_{11}{ }^{2}+\left(\omega Z_{P}+2 \bar{R}_{11}\right)^{2}}=1-E_{P}^{D}
$$

wherein we define $E_{P}{ }^{D}$ as the specific energy dissipated per unit time. In Fig. 6, we have plotted the normalized energy against incident wave frequency for a variety of asperity viscosities and interface roughness. We find that for different interface roughness, the energy dissipation reaches maxima at different asperity viscosity.

\section{SUMMARY AND CONCLUSIONS}

The main findings of this work are summarized as follows:

1. Effective stiffnesses of interfaces between two rough solids are known to be affected by surface roughness, asperity contact elasticity and viscosity. A micromechanical methodology can be used for modeling complex-valued effective stiffness accounting for asperity interaction.

2. The derived effective stiffnesses can be used to investigate plane wave propagation behavior through interfaces utilizing the imperfectly bonded interface model.

3. We find that the amplitudes of the reflected and transmitted waves are significantly influenced by the roughness and asperity viscosity. The model may thus be used to elucidate the results of wave transmission through rough interfaces.

\section{REFERENCES}

1. Kendal, K. and Tabor, D. Proc. R. Soc. London, A323, pp. 321-340, (1971).

2. Rokhlin, S.I. and Wang, Y.J. J. Acoust. Soc Am., 89, pp. 503-515, (1991).

3. Schoenberg, M. J. Acoust. Soc Am., 68, pp. 1516-1521, (1980).

4. Misra, A. J. Eng. Mech., 123, pp. 475-484, (1997).

5. Misra, A. J Geophys Res, 104, pp. 23,175-23,187, (1999).

6. Misra, A. and Marangos, O., Acta Geophysica, 56, (in print).

7. Misra, A. and Marangos, O., "Parametric Studies of Wave Propagation Through Imperfect Interfaces Using Micromechanics Based Effective Stiffness," in Review of Progress in Quantitative Nondestructive Evaluation 27B, editedby D. O. Thompson and D. E. Chimenti, AIP Conference Proceedings vol. 975, American Institute of Physics, Melville, NY, 2008, pp. 1074-1081. 